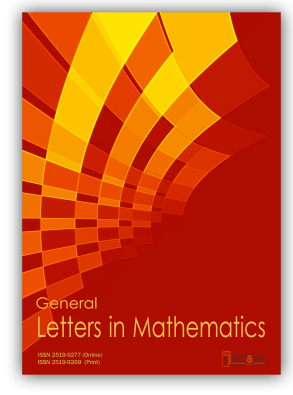

General Letters in Mathematics Vol. 3, No. 2, Oct 2017, pp.81-90

e-ISSN 2519-9277, p-ISSN 2519-9269

Available online at http:// www.refaad.com

\title{
Operational Formulae of the Multivariable Hypergeometric Matrix Functions and Related Matrix Polynomials
}

\author{
M. Abdalla ${ }^{\text {fl }}$, H. Abd-Elmageed ${ }^{2}$, \\ M. Abul-Ez ${ }^{3}$ and Z. Kishka ${ }^{4}$ \\ 1,2 Mathematics Department, Faculty of Science, South Valley University, Qena 83523, Egypt. \\ ${ }^{3,4}$ Mathematics Department, Faculty of Science, Sohag University, Sohag 82524, Egypt. \\ ${ }^{1}$ m.abdallah@sci.svu.edu.eg., ${ }^{2}$ halla_mohamed2010@yahoo.com
}

\begin{abstract}
This paper presents an overview of the modern developments around the multivariable hypergeometric matrix functions (MHMFs). We introduce operational representation of MHMFs. Also, certain classes of orthogonal matrix polynomials are established.
\end{abstract}

Keywords: Operational formulae; Generalized hypergeometric matrix functions; Orthogonal matrix polynomials; Matrix functional calculus.

MSC2010 33D15, 33C05, 15A15, 34A05.

\section{Introduction and Preliminaries}

Hypergeometric functions in one and more variables occur naturally in a wide variety of problems [4]. Various generalizations of hypergeometric functions, in particular such involving multiple functions, appear in different branches of mathematics and physics. Many of the special functions that arise in engineering, probability theory and Lie groups theory also have representations as hypergeometric functions. In recent years, in one hand, various extensions of the hypergeometric matrix functions have been presented and investigated (see, e.g., 1, 2, 12, 14, 15, 17, 18, 20, and the references cited therein). On the other hand, we may mention (see, e.g., 4, 5, 6, 9, 10, 13, 16]) who have contributed to the study of the operational formulae of some special functions. Indeed, the use of operational formulae, currently exploited in the theory of algebraic decomposition of exponential operators, may significantly simplify the study of hypergeometric matrix functions and the discovery of new relations, hardly achievable by conventional means. Taking account of operational formulae and owing to the techniques used in 13, we introduce in the present work, operator representations of multivariable hypergeometric matrix functions (MHMFs) and certain orthogonal matrix polynomials. The structure of this paper is as follows. Section 2, we introduce the definitions and properties of the generalized hypergeometric matrix functions and matrix polynomials in terms of hypergeometric matrix function. Operator representations of multivariable hypergeometric matrix functions and their miscellaneous formulas are deduced in section 3. The operational formulae of several orthogonal matrix polynomials are established in section 4 . The results obtained are believed to be new.

Now, we will summarize basic concepts and notations that will be largely exploited in this work.

Let $\mathcal{M}_{d}(\mathbb{C})$ denotes the complex vector space constituted of all square matrices with $d$ rows and $d$ columns with entries in complex space. For any matrix $M \in \mathcal{M}_{d}(\mathbb{C}), \sigma(M)$ (spectrum of M) denotes the set of all eigenvalues of

${ }^{*}$ Corresponding author. M. Abdalla ${ }^{1} \mathrm{~m}$.abdallah@sci.svu.edu.eg. 
M,

$$
\mu(M)=\max \{\operatorname{Re}(z): z \in \sigma(M)\}, \widetilde{\mu}(M)=\min \{\operatorname{Re}(z): z \in \sigma(M)\},
$$

where $\mu(M)$ is referred to as the spectral abscissa (the largest of the real parts of its eigenvalues) and $\mu(-M)=$ $-\widetilde{\mu}(M)$. The square matrix $M$ is said to be positive stable if and only if $\widetilde{\mu}(M)>0$. I and $\mathbf{0}$ stand for the identity matrix and the zero matrix in $\mathcal{M}_{d}(\mathbb{C})$, respectively.

If $f_{1}(z)$ and $f_{2}(z)$ are holomorphic functions of the complex variable $z$, which are defined in an open set $\Omega$ of the complex plane, and $M$ is a matrix in $\mathcal{M}_{d}(\mathbb{C})$ with $\sigma(M) \subset \Omega$, then from the properties of the matrix functional calculus,(cf. [3, 7, 15]) we have

$$
f_{1}(M) f_{2}(M)=f_{2}(M) f_{1}(M)
$$

Hence, if $N$ in $\mathcal{M}_{d}(\mathbb{C})$ is a matrix for which $\sigma(N) \subset \Omega$ also, and if $M N=N M$, then

$$
f_{1}(M) f_{2}(N)=f_{2}(N) f_{1}(M) .
$$

For $M \in \mathcal{M}_{d}(\mathbb{C})$ the matrix version of the Pochhammer symbol (the shifted factorial) is (cf. [3, 17, 15])

$$
(M)_{n}=M(M+I) \ldots(M+(n-1) I), \quad n \geq 1 ; \quad(M)_{0} \equiv I .
$$

The reciprocal scalar Gamma function denoted by $\Gamma^{-1}(z)=\frac{1}{\Gamma(z)}$ is an entire function of the complex variable $z$. Thus, for any $M \in \mathcal{M}_{d}(\mathbb{C})$, Riesz-Dunford functional calculus [7, 15] shows that $\Gamma^{-1}(M)$ is well defined and is, indeed, the inverse of $\Gamma(M)$. Furthermore, if

$$
M+n I \text { is invertible for all integer } n \geq 0,
$$

then

$$
(M)_{n}=\Gamma(M+n I) \Gamma^{-1}(M) .
$$

From the Notation 2.2 of [3, pp. 1007], we have

$$
\frac{(-1)^{k}}{(n-k) !} I=\frac{(-n)_{k}}{n !} I=\frac{(-n I)_{k}}{n !} ; 0 \leq k \leq n \text {. }
$$

In 1731, Euler defined the derivative formula

$$
D_{x}^{\nu} x^{\alpha}=\frac{\Gamma(\alpha+\nu)}{\Gamma(\alpha-\nu+1)} x^{\alpha-\nu}, \quad D_{x} \equiv \frac{d}{d x},
$$

where $\alpha$ and $\nu$ are arbitrary complex numbers. By application of the matrix functional calculus to this definition, for any matrix $M \in \mathcal{M}_{d}(\mathbb{C})$, one gets (see [3, 7])

$$
D_{t}^{n}\left[t^{M+m I}\right]=(M+I)_{m}\left[(M+I)_{m-n}\right]^{-1} t^{M+(m-n) I}, \quad n=0,1,2,3, \ldots,
$$

where $D_{t}=\frac{d}{d t}$ denotes the differentiation operator. Again, we observe that

$$
t^{M}\left(-D_{t}\right)^{n} t^{-M}=(M)_{n} t^{-n}
$$

and

$$
t^{I-M}\left(D_{t}^{-1}\right)^{n} t^{M-I}=\left[(M)_{n}\right]^{-1} t^{n},
$$

where $D_{t}^{-1}=\frac{1}{D_{t}}$ is the inverse operator (primitive operator) of $D_{t}$.

On other hand, if $M \in \mathcal{M}_{d}(\mathbb{C})$, and $z$ is any complex number, then the matrix exponential $e^{M z}$ is defined to be

$$
\begin{gathered}
e^{M z}=I+M z+\ldots+\frac{M^{n}}{n !} z^{n}+\ldots, \\
\frac{d^{n}}{d z^{n}}\left[e^{M z}\right]=M^{n} e^{M z}=e^{M z} M^{n}, \quad n=0,1,2,3, \ldots
\end{gathered}
$$




\section{Definitions and Miscellaneous Formulas}

In this section, we give the definitions of the following generalized hypergeometric matrix function and certain matrix polynomials in terms of hypergeometric matrix function. (see [17, 19]). Let $p$ and $q$ be finite positive integers, then the generalized hypergeometric matrix function has been given by the matrix power series as the following

$$
F\left(A_{i}, B_{j} ; z\right)=\sum_{n \geq 0} \prod_{i=1}^{p}\left(A_{i}\right)_{n} \prod_{j=1}^{q}\left[\left(B_{j}\right)_{n}\right]^{-1} \frac{z^{n}}{n !} .
$$

For commutative matrices $A_{i}, \quad 1 \leq i \leq p$ and $B_{j}, \quad 1 \leq j \leq q$ in $\mathcal{M}_{d}(\mathbb{C})$ such that

$$
B_{j}+n I \text { are invertible for all integers } n \geq 0 \text {. }
$$

The following results may be proved using the standard techniques of convergence theory:

- If $p \leq q$, then the power series (2.1) is convergence for all finite $z$.

- If $p=q+1$, then the power series (2.1) is convergent for $|z|<1$ and diverges for $|z|>1$.

- If $p>q+1$, then the power series (2.1) diverges for $z, z \neq 0$.

- If $p=q+1$, then the power series (2.1) is absolutely convergent on the circle $|z|=1$ when

$$
\sum_{i=0}^{p} \widetilde{\mu}\left(A_{i}\right)<\sum_{j=0}^{q} \mu\left(B_{j}\right)
$$

where $\widetilde{\mu}\left(A_{i}\right)$ and $\mu\left(B_{j}\right)$ as defined in (1.1).

With $p=1$ and $q=0$ in (2.1), one gets the following relation due to [15]

$$
(1-z)^{-A}=\sum_{n=0}^{\infty}(A)_{n} \frac{z^{n}}{n !}, \quad|z|<1,
$$

while at $p=1$ and $q=1$ in $(2.1)$, we have

$$
e^{z}=\sum_{n=0}^{\infty} \frac{z^{n}}{n !}
$$
(cf. [15])

Also, with three matrix parameter; $p=2$ and $q=1$ in (2.1) we have the Gauss matrix function in the form

$$
{ }_{2} F_{1}\left(A_{1}, A_{2} ; B_{1} ; z\right)=\sum_{n=0}^{\infty}\left(A_{1}\right)_{n}\left(A_{2}\right)_{n}\left[\left(B_{1}\right)_{n}\right]^{-1} \frac{z^{n}}{n !},
$$

where $A_{1}, A_{2}$ and $B_{1}$ are matrices in $\mathcal{M}_{d}(\mathbb{C})$, and $B_{1}$ satisfying the condition (1.2). (see [7, 21]). For an arbitrary matrix $A \in \mathcal{M}_{d}(\mathbb{C})$, with $A+n I$ invertible for every integer $n \geq 1$, then the $\mathrm{n}$-th Laguerre matrix polynomials $L_{n}^{A}(z)$ is defined by

$$
L_{n}^{A}(z)=\frac{(A+I)_{n}}{n !}{ }_{1} F_{1}(-n I ; A+I ; z) .
$$

therefore, we can be reduced to Shively's pseudo Laguerre matrix polynomial in the form

$$
R_{n}^{A}(z)=\frac{(A)_{2 n}\left[(A)_{n}\right]^{-1}}{n !}{ }_{1} F_{1}(-n I ; A+I ; z) .
$$

(see [11]). Let $A$ and $B$ be parameter commuting matrices in $\mathcal{M}_{d}(\mathbb{C})$ satisfying the spectral condition (1.2). For any natural number $n \geq 0$, the $\mathrm{n}$-th generalized Bessel matrix polynomial $\mathcal{Y}_{n}^{(A, B)}(z)$ is defined by

$$
\mathcal{Y}_{n}^{(A, B)}(z)={ }_{2} F_{0}\left(-n I, A+(n-1) I ;-; z B^{-1}\right) .
$$


Also, the n-th reverse generalized Bessel matrix polynomial $\Theta_{n}^{(A, B)}(z)$ is defined in [3] by

$$
\begin{aligned}
\Theta_{n}^{(A, B)}(z) & =(-1)^{n} \Gamma^{-1}(-A-(2 n-2) I) \Gamma(-A+(n-2) I) \\
& \times{ }_{1} F_{1}(-n I ;-A-(2 n-2) I ; B z) .
\end{aligned}
$$

(see [8, 24]). Suppose that $A$ and $B$ are positive stable matrices in $\mathcal{M}_{d}(\mathbb{C})$ satisfying the condition

$$
\operatorname{Re}(a)>-1, \quad \forall a \in \sigma(A) \text { and } \operatorname{Re}(a)>-1, \quad \forall b \in \sigma(B) \text {. }
$$

For any positive integer $\mathrm{n}$, the Jacobi matrix polynomials $P_{n}^{A, B}(z)$ are defined by

$$
P_{n}^{A, B}(z)=\frac{(A+I)_{n}}{n !}{ }_{2} F_{1}\left(-n I, A+B+(n+1) I ; A+I ; \frac{1-z}{2}\right) .
$$

The special case of $A=B$ of the Jacobi matrix polynomial is called ultraspherical matrix polynomial and is denoted by $P_{n}^{A, A}(z)$. It is thus defined as

$$
P_{n}^{A, A}(z)=\frac{(A+I)_{n}}{n !}{ }_{2} F_{1}\left(-n I, 2 A+(n+1) I ; A+I ; \frac{1-z}{2}\right) .
$$

(see [22]). The Gegenbauer matrix polynomials $C_{n}^{A}(z)$ is defined in the form

$$
C_{n}^{A}(z)=\frac{(2 A)_{n}}{n !}{ }_{2} F_{1}\left(-n I, 2 A+n I ; A+\frac{1}{2} I ; \frac{1-z}{2}\right),
$$

where $A$ is a positive stable matrix in $\mathcal{M}_{d}(\mathbb{C})$. With $d=1$ and $A=\frac{1}{2}$, we define the class of orthogonal Legendre polynomials in the form

$$
P_{n}(z)={ }_{2} F_{1}\left(-n,(n+1) ; \frac{1-z}{2}\right)
$$

(see [19]). The generalized Bateman's matrix polynomials $Z_{n}^{A, B}(z)$ is defined in the form

$$
Z_{n}^{A, B}(z)={ }_{2} F_{2}\left(-n I, 2 A+n I ; A+\frac{1}{2} I, B+I ; z\right),
$$

where $A$ and $B$ are commutative matrices in $\mathcal{M}_{d}(\mathbb{C})$ and $A+n I$ and $B+n I$ are invertible for all integer $n \geq 0$. (see [23]). The Rices matrix polynomials $H_{n}^{A, B}(z)$ is defined by means of the relation

$$
H_{n}^{A, B}(z)={ }_{3} F_{2}(-n I,(n+1) I, A ; I, B ; z),
$$

for commutative matrices $A$ and $B$ in $\mathcal{M}_{d}(\mathbb{C})$ and $B$ satisfying the condition (1.2).

\section{Operational Formulae of The MHMFs}

In this section, our main theorem is stated as follows: Suppose that $M, A_{1}, A_{2}, \ldots, A_{p}$ and $B_{1}, B_{2}, \ldots, B_{q}$ are parameter commuting matrices in $\mathbb{C}^{N \times N}$ while $B_{1}, B_{2}, \ldots, B_{q}$ satisfying the spectral condition $(2.2)$ and let $D_{z_{\mu}}=\frac{\partial}{\partial z_{\mu}}$ and $D_{w_{\mu}}=\frac{\partial}{\partial w_{\mu}} ; \quad \mu=1,2,3, \ldots$, be partial differential operators; $z_{1}, z_{2}, \ldots, z_{q}$ and $w_{1}, w_{2}, \ldots, w_{p} \in \mathbb{C}$ then, we have the following operational representations

$$
\begin{aligned}
& { }_{p+1} F_{q}\left(M, A_{1}, A_{2}, \ldots, A_{p} ; B_{1}, B_{2}, \ldots, B_{q} ; \frac{z_{1}, z_{2}, \ldots, z_{q}}{w_{1}, w_{2}, \ldots, w_{p}}\right) \\
= & \prod_{r=1}^{p} w_{r}^{A_{r}} \prod_{s=1}^{q} z_{s}^{-B_{s}+I}\left(1-\frac{(-1)^{p} \prod_{r=1}^{p} D_{w_{r}}}{\prod_{s=1}^{q} D_{z_{s}}}\right)^{-M} \prod_{r=1}^{p} w_{r}^{-A_{r}} \prod_{s=1}^{q} z_{s}^{B_{s}-I}
\end{aligned}
$$


and

$$
\begin{aligned}
& { }_{p} F_{q}\left(A_{1}, A_{2}, \ldots, A_{p} ; B_{1}, B_{2}, \ldots, B_{q} ; \frac{z_{1}, z_{2}, \ldots, z_{q}}{w_{1}, w_{2}, \ldots, w_{p}}\right) \\
= & \prod_{r=1}^{p} w_{r}^{A_{r}} \prod_{s=1}^{q} z_{s}^{-B_{s}+I} \exp \left\{\frac{(-1)^{p} \prod_{r=1}^{p} D_{w_{r}}}{\prod_{s=1}^{q} D_{z_{s}}}\right\} \prod_{r=1}^{p} w_{r}^{-A_{r}} \prod_{s=1}^{q} z_{s}^{B_{s}-I} .
\end{aligned}
$$

Proof. Applying the result (2.3) in the right hand side of (3.1), it follows that

$$
\begin{aligned}
& \prod_{r=1}^{p} w_{r}^{A_{r}} \prod_{s=1}^{q} z_{s}^{-B_{s}+I}\left(1-\frac{(-1)^{p} \prod_{r=1}^{p} D_{w_{r}}}{\prod_{s=1}^{q} D_{z_{s}}}\right)^{-M} \prod_{r=1}^{p} w_{r}^{-A_{r}} \prod_{s=1}^{q} z_{s}^{B_{s}-I} \\
= & \left.\prod_{r=1}^{p} w_{r}^{A_{r}} \prod_{s=1}^{q} z_{s}^{-B_{s}+I} \sum_{k=0}^{\infty} \frac{(M)_{k}(-1)^{k p} \prod_{r=1}^{p}\left(D_{w_{r}}\right)^{k}}{k ! \prod_{s=1}^{q}\left(D_{z_{s}}\right)^{k}}\right\} \prod_{r=1}^{p} w_{r}^{-A_{r}} \prod_{s=1}^{q} z_{s}^{B_{s}-I} \\
= & \sum_{k=0}^{\infty} \frac{(M)_{k}}{k !} \prod_{r=1}^{p}\left\{w_{r}^{A_{r}}\left(-D_{w_{r}}\right)^{k} w_{r}^{-A_{r}}\right\} \prod_{s=1}^{q}\left\{z_{s}^{-B_{s}+I}\left(\frac{1}{D_{z_{s}}}\right)^{k} z_{s}^{B_{s}-I}\right\} .
\end{aligned}
$$

According to the relations (1.4) and (1.5), we obtain

$$
\begin{aligned}
& \prod_{r=1}^{p} w_{r}^{A_{r}} \prod_{s=1}^{q} z_{s}^{-B_{s}+I}\left(1-\frac{(-1)^{p} \prod_{r=1}^{p} D_{w_{r}}}{\prod_{s=1}^{q} D_{z_{s}}}\right)^{-M} \prod_{r=1}^{p} w_{r}^{-A_{r}} \prod_{s=1}^{q} z_{s}^{B_{s}-I} \\
= & \sum_{k=0}^{\infty} \frac{(M)_{k}}{k !} \prod_{r=1}^{p}\left\{w_{r}^{-k}\left(A_{r}\right)_{k}\right\} \prod_{s=1}^{q}\left\{z_{s}^{k}\left[\left(B_{s}\right)_{k}\right]^{-1}\right\} .
\end{aligned}
$$

Hence, from (2.1), we get the asserted result according to (3.1).

Taking the right hand side of (3.2) with the definition (2.4), we have

$$
\begin{aligned}
& \prod_{r=1}^{p} w_{r}^{A_{r}} \prod_{s=1}^{q} z_{s}^{-B_{s}+I} \exp \left\{\frac{(-1)^{p} \prod_{r=1}^{p} D_{w_{r}}}{\prod_{s=1}^{q} D_{z_{s}}}\right\} \prod_{r=1}^{p} w_{r}^{-A_{r}} \prod_{s=1}^{q} z_{s}^{B_{s}-I} \\
= & \left.\prod_{r=1}^{p} w_{r}^{A_{r}} \prod_{s=1}^{q} z_{s}^{-B_{s}+I} \sum_{k=0}^{\infty} \frac{(-1)^{k p} \prod_{r=1}^{p}\left(D_{w_{r}}\right)^{k}}{k ! \prod_{s=1}^{q}\left(D_{z_{s}}\right)^{k}}\right\} \prod_{r=1}^{p} w_{r}^{-A_{r}} \prod_{s=1}^{q} z_{s}^{B_{s}-I} \\
= & \sum_{k=0}^{\infty} \frac{1}{k !} \prod_{r=1}^{p}\left\{w_{r}^{A_{r}}\left(-D_{w_{r}}\right)^{k} w_{r}^{-A_{r}}\right\} \prod_{s=1}^{q}\left\{z_{s}^{-B_{s}+I}\left(\frac{1}{D_{z_{s}}}\right)^{k} z_{s}^{B_{s}-I}\right\} .
\end{aligned}
$$

According to the relations (1.4) and (1.5), we obtain

$$
\begin{aligned}
& \prod_{r=1}^{p} w_{r}^{A_{r}} \prod_{s=1}^{q} z_{s}^{-B_{s}+I} \exp \left\{\frac{(-1)^{p} \prod_{r=1}^{p} D_{w_{r}}}{\prod_{s=1}^{q} D_{z_{s}}}\right\} \prod_{r=1}^{p} w_{r}^{-A_{r}} \prod_{s=1}^{q} z_{s}^{B_{s}-I} \\
= & \sum_{k=0}^{\infty} \frac{1}{k !} \prod_{r=1}^{p}\left\{w_{r}^{-k}\left(A_{r}\right)_{k}\right\} \prod_{s=1}^{q}\left\{z_{s}^{k}\left[\left(B_{s}\right)_{k}\right]^{-1}\right\} .
\end{aligned}
$$

Again, from (2.1), we have proved the relation (3.2) in our main theorem.

Putting different values of $p$ and $q$ in (3.1) and (3.2), we obtain operational representations of MHMFs.

If we take $p=1$ and $q=0$ in (3.2), we see

$$
{ }_{1} F_{0}\left(A ;-; \frac{1}{z}\right)=z^{A} \exp \left(D_{z}\right) z^{-A}
$$


Similarly, we present the following results:

$$
\begin{aligned}
& { }_{1} F_{1}\left(A ; B ; \frac{z}{w}\right)=w^{A} z^{-B+I} \exp \left(\frac{-D_{w}}{D_{z}}\right) w^{-A} z^{B-I} . \\
& { }_{1} F_{1}(A ; B ; z)=z^{-B+I}\left(1+\frac{1}{D_{z}}\right)^{-A} z^{B-I} . \\
& { }_{2} F_{0}\left(A, B ; \frac{1}{z}\right)=z^{A}\left(1+D_{z}\right)^{-B} z^{-A} . \\
& { }_{2} F_{0}\left(A, B ; \frac{1}{z w}\right)=z^{A} w^{B} \exp \left(D_{z} D_{w}\right) z^{-A} w^{-B} . \\
& { }_{2} F_{1}\left(A_{1}, A_{2} ; B_{1} ; \frac{z}{w}\right)=z^{-B_{1}+I} w^{A_{2}}\left(1+\frac{D_{w}}{D_{z}}\right)^{-A_{1}} z^{B_{1}-I} w^{-A_{2}} . \\
& { }_{2} F_{1}\left(A_{1}, A_{2} ; B_{1} ; \frac{z}{w}\right)=z^{-B_{1}+I} w^{A_{1}}\left(1+\frac{D_{w}}{D_{z}}\right)^{-A_{2}} z^{B_{1}-I} w^{-A_{1}} . \\
& { }_{2} F_{1}\left(A_{1}, A_{2} ; B_{1} ; \frac{z}{w_{1} w_{2}}\right)=z^{-B_{1}+I} w_{1}^{A_{1}} w_{2}^{A_{2}} \exp \left(\frac{D_{w_{1}} D_{w_{2}}}{D_{z}}\right) z^{B_{1}-I} w_{1}^{-A_{1}} w_{2}^{-A_{2}} \\
& { }_{2} F_{2}\left(A_{1}, A_{2} ; B_{1}, B_{2} ; \frac{z_{1} z_{2}}{w}\right)=z_{1}^{-B_{1}+I} z_{2}^{-B_{2}+I} w^{A_{2}}\left(1+\frac{D_{w}}{D_{z_{1}} D_{z_{2}}}\right)^{-A_{1}} z_{1}^{B_{1}-I} z_{2}^{B_{2}-I} w^{-A_{2}} . \\
& { }_{2} F_{2}\left(A_{1}, A_{2} ; B_{1}, B_{2} ; \frac{z_{1} z_{2}}{w}\right)=z_{1}^{-B_{1}+I} z_{2}^{-B_{2}+I} w^{A_{1}}\left(1+\frac{D_{w}}{D_{z_{1}} D_{z_{2}}}\right)^{-A_{2}} z_{1}^{B_{1}-I} z_{2}^{B_{2}-I} w^{-A_{1}} . \\
& { }_{2} F_{2}\left(A_{1}, A_{2} ; B_{1}, B_{2} ; \frac{z_{1} z_{2}}{w}\right)=z_{1}^{-B_{2}+I} z_{2}^{-B_{1}+I} w^{A_{2}}\left(1+\frac{D_{w}}{D_{z_{1}} D_{z_{2}}}\right)^{-A_{1}} z_{1}^{B_{2}-I} z_{2}^{B_{1}-I} w^{-A_{2}} \\
& { }_{2} F_{2}\left(A_{1}, A_{2} ; B_{1}, B_{2} ; \frac{z_{1} z_{2}}{w_{1} w_{2}}\right)=z_{1}^{-B_{2}+I} z_{2}^{-B_{1}+I} w_{1}^{A_{1}} w_{2}^{A_{2}} \exp \left(\frac{D_{w_{1}} D_{w_{2}}}{D_{z_{1}} D_{z_{2}}}\right) \\
& \times z_{1}^{B_{2}-I} z_{2}^{B_{1}-I} w_{1}^{-A_{1}} w_{2}^{-A_{2}} . \\
& { }_{2} F_{2}\left(A_{1}, A_{2} ; B_{1}, B_{2} ; \frac{z_{1} z_{2}}{w_{1} w_{2}}\right)=z_{1}^{-B_{1}+I} z_{2}^{-B_{2}+I} w_{1}^{A_{1}} w_{2}^{A_{2}} \exp \left(\frac{D_{w_{1}} D_{w_{2}}}{D_{z_{1}} D_{z_{2}}}\right) \\
& \times z_{1}^{B_{1}-I} z_{2}^{B_{2}-I} w_{1}^{-A_{1}} w_{2}^{-A_{2}} . \\
& { }_{3} F_{0}\left(A_{1}, A_{2}, A_{3} ;-; \frac{1}{w_{1} w_{2}}\right)=w_{2}^{A_{2}} w_{1}^{A_{3}}\left(1-D_{w_{1}} D_{w_{2}}\right)^{-A_{1}} w_{2}^{-A_{2}} w_{1}^{-A_{3}} \\
& { }_{3} F_{0}\left(A_{1}, A_{2}, A_{3} ;-; \frac{1}{w_{1} w_{2}}\right)=w_{2}^{A_{1}} w_{1}^{A_{3}}\left(1-D_{w_{1}} D_{w_{2}}\right)^{-A_{2}} w_{2}^{-A_{1}} w_{1}^{-A_{3}} \\
& { }_{3} F_{0}\left(A_{1}, A_{2}, A_{3} ;-; \frac{1}{w_{1} w_{2}}\right)=w_{2}^{A_{1}} w_{1}^{A_{2}}\left(1-D_{w_{1}} D_{w_{2}}\right)^{-A_{3}} w_{2}^{-A_{1}} w_{1}^{-A_{2}}
\end{aligned}
$$




$$
\begin{gathered}
{ }_{3} F_{0}\left(A_{1}, A_{2}, A_{3} ;-; \frac{1}{w_{1} w_{2} w_{3}}\right)=w_{2}^{A_{1}} w_{1}^{A_{2}} w_{3}^{A_{3}} ; \exp \left(D_{w_{1}} D_{w_{2}} D_{w_{3}}\right) w_{2}^{-A_{1}} w_{1}^{-A_{2}} w_{3}^{-A_{3}} . \\
{ }_{3} F_{1}\left(A_{1}, A_{2}, A_{3} ; B_{1} ; \frac{z}{w_{1} w_{2}}\right)=z^{-B_{1}+I} w_{1}^{A_{1}} w_{2}^{A_{2}}\left(1-\frac{D_{w_{1}} D_{w_{2}}}{D_{z}}\right)^{-A_{3}} z^{B_{1}-I} w_{1}^{-A_{1}} w_{2}^{-A_{2}} \\
{ }_{3} F_{1}\left(A_{1}, A_{2}, A_{3} ; B_{1} ; \frac{z}{w_{1} w_{2}}\right)=z^{-B_{1}+I} w_{1}^{A_{1}} w_{2}^{A_{3}}\left(1-\frac{D_{w_{1}} D_{w_{2}}}{D_{z}}\right)^{-A_{2}} z^{B_{1}-I} w_{1}^{-A_{1}} w_{2}^{-A_{3}} . \\
{ }_{3} F_{1}\left(A_{1}, A_{2}, A_{3} ; B_{1} ; \frac{z}{w_{1} w_{2}}\right)=z^{-B_{1}+I} w_{1}^{A_{2}} w_{2}^{A_{3}}\left(1-\frac{D_{w_{1}} D_{w_{2}}}{D_{z}}\right)^{-A_{1}} z^{B_{1}-I} w_{1}^{-A_{2}} w_{2}^{-A_{3}} . \\
{ }_{3} F_{1}\left(A_{1}, A_{2}, A_{3} ; B_{1} ; \frac{z}{w_{1} w_{2} w_{3}}\right)=z^{-B_{1}+I} w_{1}^{A_{2}} w_{2}^{A_{3}} w_{3}^{A_{1}} \\
\exp \left(\frac{-D_{w_{1}} D_{w_{2}} D_{w_{3}}}{D_{z}}\right) z^{B_{1}-I} w_{1}^{-A_{2}} w_{2}^{-A_{3}} w_{3}^{-A_{1}}
\end{gathered}
$$

\section{Some Basic Applications}

In the present section, we give the operational representations of many orthogonal matrix polynomials as follows

$$
\begin{gathered}
L_{n}^{A}(z)=\frac{z^{-A}}{n !}\left(D_{z}-1\right)^{n} z^{A+n I}, \\
L_{n}^{A}(z)=\frac{(A+I)_{n}}{n !} z^{-A}\left(1-\frac{1}{D_{z}}\right)^{n} z^{A}, \\
L_{n}^{A}\left(\frac{z}{w}\right)=\frac{(A+I)_{n}}{n !} z^{-A} w^{-n} \exp \left(\frac{-D_{w}}{D_{z}}\right) z^{A} w^{n}, \\
R_{n}^{A}(z)=\frac{(A)_{2 n}\left[(A)_{n}\right]^{-1}}{n !} z^{-A+(1-n) I}\left(1+\frac{1}{D_{z}}\right)^{n} z^{A+(n-1) I} . \\
R_{n}^{A}\left(\frac{z}{w}\right)=\frac{(A)_{2 n}\left[(A)_{n}\right]^{-1}}{n !} z^{-A+(1-n) I} w^{-n} \exp \left(\frac{-D_{w}}{D_{z}}\right) z^{A+(n-1) I} w^{n} . \\
\mathcal{Y}_{n}^{(A, B)}\left(\frac{1}{z}\right)=z^{-n}\left(1-D_{z} B^{-1}\right)^{-(A+(n-1) I)} z^{n} . \\
\mathcal{Y}_{n}^{(A, B)}\left(\frac{1}{z}\right)=z^{A+(n-1) I}\left(1-D_{z} B^{-1}\right)^{-n} z^{-(A+(n-1) I)} . \\
P_{n}^{A, B}\left(1-\frac{2 z}{w}\right)=\frac{z^{-A} w^{-n}}{n !} D_{z}^{n}\left(1+\frac{D_{w}}{D_{z}}\right)^{-(A+B+(n+1) I)} z^{A+n I} w^{n} .
\end{gathered}
$$




$$
\begin{aligned}
& P_{n}^{A, B}\left(1-\frac{2 z}{w}\right)=\frac{(A+I)_{n}}{n !} z^{-A} w^{A+B+(n+1) I}\left(1+\frac{D_{w}}{D_{z}}\right)^{n} z^{A} w^{-(A+B+(n+1) I)} . \\
& P_{n}^{A, B}\left(1-\frac{2 z}{w}\right)=\frac{(A+I)_{n}}{n !} z^{-A} w^{-n}\left(1+\frac{D_{w}}{D_{z}}\right)^{-(A+B+(n+1) I)} z^{A} w^{n} \\
& P_{n}^{A, A}\left(1-\frac{2 z}{w}\right)=\frac{z^{-A} w^{-n}}{n !} D_{z}^{n}\left(1+\frac{D_{w}}{D_{z}}\right)^{-(2 A+(n+1) I)} z^{A+n I} w^{n} . \\
& P_{n}^{A, A}\left(1-\frac{2 z}{w}\right)=\frac{(A+I)_{n}}{n !} z^{-A} w^{2 A+(n+1) I}\left(1+\frac{D_{w}}{D_{z}}\right)^{n} z^{A} w^{-(2 A+(n+1) I)} . \\
& P_{n}^{A, A}\left(1-\frac{2 z}{w}\right)=\frac{(A+I)_{n}}{n !} z^{-A} w^{-n}\left(1+\frac{D_{w}}{D_{z}}\right)^{-(2 A+(n+1) I)} z^{A} w^{n} . \\
& C_{n}^{A}\left(1-\frac{2 z}{w}\right)=\frac{(2 A)_{n}}{n !} z^{-A+\frac{1}{2} I} w^{-n}\left(1+\frac{D_{w}}{D_{z}}\right)^{-(2 A+n I)} z^{A-\frac{1}{2} I} w^{n} \\
& C_{n}^{A}\left(1-\frac{2 z}{w}\right)=\frac{(2 A)_{n}}{n !} z^{-A+\frac{1}{2} I} w^{2 A+n I}\left(1+\frac{D_{w}}{D_{z}}\right)^{n} z^{A-\frac{1}{2} I} w^{-(2 A+n I)} . \\
& Z_{n}^{A, B}\left(\frac{z_{1} z_{2}}{w_{1}}\right)=w_{1}^{2 A+n I} z_{1}^{-A+\frac{1}{2} I} z_{2}^{-B}\left(1+\frac{D_{w_{1}}}{D_{z_{1} D_{z_{2}}}}\right)^{n} \\
& w_{1}^{-2 A-n I} z_{1}^{A-\frac{1}{2} I} z_{2}^{B} \text {. } \\
& Z_{n}^{A, B}\left(\frac{z_{1} z_{2}}{w_{1}}\right)=w_{1}^{-n} z_{1}^{-B} z_{2}^{-A+\frac{1}{2} I}\left(1+\frac{D_{w_{1}}}{D_{z_{1} D_{z_{2}}}}\right)^{-(2 A+n I)} \\
& w_{1}^{n} z_{1}^{B} z_{2}^{A-\frac{1}{2} I} \\
& Z_{n}^{A, B}\left(\frac{z_{1} z_{2}}{w_{1} w_{2}}\right)=w_{2}^{(2 A+n I)} w_{1}^{-n} z_{1}^{-B} z_{2}^{-A+\frac{1}{2}} \exp \left(\frac{D_{w_{1}} D_{w_{1}}}{D_{z_{1} D_{z_{2}}}}\right) \\
& \times w_{2}^{-(2 A+n I)} w_{1}^{n} z_{1}^{B} z_{2}^{A-\frac{1}{2} I} . \\
& H_{n}^{A, B}\left(\frac{w}{z}\right)=\frac{z^{A}}{n !} D_{w}^{n}\left(w^{-B+(n+1) I}\left(1+\frac{D_{z}}{D_{w}}\right)^{n} z^{-A} w^{-(B+I)}\right) \\
& H_{n}^{A, B}\left(C, D ; \frac{z_{1} z_{2}}{w_{1} w_{2}}\right)=z_{1}^{-C} z_{2}^{-B+I} w_{1}^{-n} w_{2}^{A}\left(1-\frac{D_{w_{1}} D_{w_{1}}}{D_{z_{1} D_{z_{2}}}}\right)^{-(C+D+(n+1) I)} \\
& \times z_{1}^{C} z_{2}^{B-I} w_{1}^{n} w_{2}^{-A}
\end{aligned}
$$




\section{Outlook and Concluding Remarks}

The paper proposes to introduce operational formulae of some interesting multivariable hypergeometric matrix functions (MHMFs). The induced MHMFs which have been investigated are Laguerre matrix polynomial, generalized Bessel matrix polynomial, Jacobi matrix polynomials, Gegenbauer matrix polynomial, generalized Bateman's matrix polynomial and Rices matrix polynomial. Further research on this topic is now under investigation and will be reported in forthcoming papers concerning: Lagrange matrix polynomial, Sister Celines matrix polynomial, Bedients matrix polynomial etc.

\section{References}

[1] M. Abdalla; On the incomplete hypergeometric matrix functions, Ramanujan J., 43, (2017), 663-678.

[2] M. Abdalla and A. Bakhet; Extended Gauss hypergeometric matrix functions, Published online in Iran. J. Sci. Technol. Trans. Sci., (2017). DOI 10.1007/s40995-017-0183-3.

[3] M. A. Abul-Dahab, M. Abul-Ez, Z. Kishka and D. Constales; Reverse generalized Bessel matrix differential equation, polynomial solutions, and their properties, Math. Methods. Appl. Sci., 38, (2015), 1005-1013.

[4] M. G. Bin-Saad; Symbolic operational images and decomposition formulas of hypergeometric functions, J. Math. Anal. Appl., 376, (2011), 451-468.

[5] M. G. Bin-Saad and A. Hasanov; Linear independent solutions and operational representations for hypergeometric functions of four variables, Chinese J. Math., 2014. http://dx.doi.org/10.1155/2014/273064

[6] M. G. Bin-Saad and M. A. Hussein, Operational images and relations of two and three variable hypergeometric series, J. Progr. Res. Math., 2, (2015), 39-46.

[7] L. Jódar and J. Sastre; On the Laguerre matrix polynomials, Utilitas Math., 53, (1998), 37-48.

[8] E. Defez, L. Jódar and A. Law; Jacobi matrix differential equation, polynomial solutions, and their properties, Comput. Math. Appl., 48, (2004), 789-803.

[9] A. Ghanmi; Operational formulae for the complex Hermite polynomials $H_{p, q}(z, \bar{z})$, Integral Transforms Spec. Funct., 24 (2013), 884-895.

[10] M. E. Ismail; Analytic properties of complex Hermite polynomials, Trans. Amer. Math. Soci., 368, (2016), 1189-1210.

[11] Z. M. Kishka, A. Shehata and M. Abul-Dahab; The generalized Bessel matrix polynomials, J. Math. Comput. Sci., 2, (2012), 305-316.

[12] Z. M. Kishka, A. Shehata and M. Abul-Dahab; A new extension of hypergeometric matrix functions, Advan. Appl. Math. Sci., 10, (2011), 349-371.

[13] M. A. Khan and M. K. Khan; Operator representations of generalized hypergeometric functions and certain polynomials, Mat. Vesnik., 63, (2011), 253-262.

[14] L. Jódar and J.C. Cortés; Closed form general solution of the hypergeometric matrix differential equation, Math. Computer Modell., 32, (2000), 1017-1028.

[15] L. Jódar and J.C. Cortés; On the hypergeometric matrix function, J. Comp. Appl. Math., 99, (1998), $205-217$.

[16] K. Maleknejad, K. Nouri and L. Torkzadeh; Operational matrix of fractional integration based on the Shifted second kind Chebyshev polynomials for solving fractional differential equations., Mediterr. J. Math., 13, (2016), 1377-1390.

[17] K. A. M. Sayyed, M. S. Metwally and M. T. Mohammed; Certain hypergeometric matrix function, Scien. Math. Japon., 69, (2009), 315-321.

[18] A. Salem, The basic Gauss hypergeometric matrix function and its matrix q-difference equation, Linear and Multilinear Algebra., 62, (2014), 347-361.

[19] A. Shehata; Some relations on Konhauser matrix polynomials, Misko. Math. Not., 17, (2016), 605-633. 
[20] A. Shehata; New kinds of hypergeometric matrix functions, British J. Math. and Comput. Sci., 5, (2015), 92-103.

[21] A. Shehata; Some relations on Laguerre matrix polynomials, Malays. J. Math. Sci., 9, (2015), 443-462.

[22] A. Shehata; Some relations on Gegenbauer matrix polynomials, Revi. Compu. Engin. Resea., 2, (2015), 1-21.

[23] A. Shehata; On Rice's matrix polynomials, Afr. Matem., 25, (2014), 757-777.

[24] F. Taşdelena ,B. Çekimb and R. Aktaş; On a multivariable extension of Jacobi matrix polynomials, Comput. Math. Appl., 61, (2011), 2412-2423. 